\title{
ArcheoSciences
}

Revue d'archéométrie

\section{Identification de résines végétales datant de l'Egypte ancienne par Chromatographie en phase gazeuse couplée à un spectromètre de masse}

Carole Mathe, Michael Hovaneissian, Paul Archier et Catherine Vieillescazes

\section{(e) OpenEdition}

Journals

Édition électronique

URL : http://journals.openedition.org/archeosciences/658

DOI : $10.4000 /$ archeosciences.658

ISBN : 978-2-7535-1594-9

ISSN : $2104-3728$

\section{Éditeur}

Presses universitaires de Rennes

\section{Édition imprimée}

Date de publication : 31 décembre 2005

Pagination : 157-161

ISSN : 1960-1360

\section{Référence électronique}

Carole Mathe, Michael Hovaneissian, Paul Archier et Catherine Vieillescazes, «Identification de résines végétales datant de l'Egypte ancienne par Chromatographie en phase gazeuse couplée à un spectromètre de masse », ArcheoSciences [En ligne], 29 | 2005, mis en ligne le 31 décembre 2007, consulté le 11 février 2021. URL : http://journals.openedition.org/archeosciences/658 ; DOI : https:// doi.org/10.4000/archeosciences.658 


\title{
Identification de résines végétales datant de l'Egypte ancienne par chromatographie en phase gazeuse couplée à un spectromètre de masse
}

\author{
Carole MATHE*, Michael HOVANEISSIAN*, Paul ARCHIER* et Catherine VIEILLESCAZES*
}

\begin{abstract}
Résumé : Trois échantillons, respectivement référencés L24, L42 et L36 provenant de la collection Victor Loret de l'Institut d'Egyptologic (Université Lyon II, Pr J.-C. Goyon), ont été analysés. Ils ont été prélevés lors de fouilles effectuées par l'archéologuc J. de Morgan sur lc sitc de Dashour dans les années 1894 et 1895 . L'analyse de ces matériaux, menéc par Chromatographic en Phase Gazcuse coupléc à un Spectromètre de Masse (CPG/SM), a mis en évidence l'existence de véritables formulations. Le prélèvement L24 a été identifié comme étant un mélange constitué d'une résine appartenant à la famille des Ombellifères et plus précisément au genre Ferula, d'un corps gras ainsi que d'une résine de conifêre.
\end{abstract}

Abstract: Threc samples, respectively referred L24, L42 and L36 coming from the collection Victor Loret of the Egyptologic Institute (University Lyon II, Pr J.-C. Goyon), were analyzed. They were recovered from excavations at Dashour in 1894-1895 by the archacologist J. de Morgan. The analysis of these materials carried out by Gas Chromatography coupled to a Mass Spectrometer (GC/MS) and the results show the existence of real formulations. L24 was identified as a mixture of a resin belonging to the family of Umbellifereae and more precisely to the Ferula genus, a fatty substance and a resin of conifer.

Mots-clés : CPG/SM, résine naturelle, ombelliférone, diterpènes, égyptologic.

Key-words: (iC/MS, natural resin, umbelliferone, diterpenoids, egyptology.

\section{Introduction}

La mise en évidence de matériaux organiques résineux dans des échantillons archéologiques peut fournir de précieux renseignements quant à l'utilisation ancestrale des produits naturels lors de cérémonies rituelles ou en tant que colles, adhésifs, parfums, cosmétiques, etc. La stabilité chimique de ces matériaux les rend particulièrement intéressants lors de l'analyse de prélèvements archéologiques de nature organique. Les marqueurs biochimiques qui composent ces résines permettent souvent de définir leur origine botanique.

Les résines végétales sont des matériaux complexes, pouvant être constituées de trois familles distinctes de composés. Les huiles essentielles se composent de molécules volatiles de type mono- et sesquiterpènes. La fraction résinique rassemble des composés terpéniques (solubles dans l'alcool) à vingt atomes de carbones (diterpènes), ou en $\mathrm{C}_{30}$ (triterpènes) ; enfin la partie gomme est caractérisée par des polysaccharides (hydrosolubles). Diverses catégories de substances résineuses se distinguent alors : résines, gommo-résines et oléo-gommo-résines.

Parmi les matériaux résineux, souvent complexes et hétérogènes, les dérivés diterpéniques et triterpéniques sont plus particulièrement analysés aussi bien dans des échantillons contemporains qu'au sein de prélèvements archéologiques. D'une part, ces composés s'avèrent souvent spécifiques d'une famille ou d'une espèce botanique (biomarqueurs), d'autre part sous l'action de facteurs naturels (vieillissement) ou anthropiques (chauffage), ils constituent des marqueurs potentiels de dégradation.

Parmi les substances odorantes utilisées dans le monde antique, le galbanum (Ferula galbaniflua) et la gomme ammoniaque appartenant tous deux à la famille des Ombellifères sont souvent cités dans la littérature spécialisée. Sous l'appellation de gomme ammoniaque, sont

\footnotetext{
* Laboratoire de Chimie Bioorganique et des Systèmes Moléculaires Vectoriels, Faculté des Sciences, Université d'Avignon, 33 rue Louis Pasteur, 84000 AVIGNON.

carole.mathe(a)univ-avignon.fr

michael.hovaneissian@univ-avignon.fr

paul.archier(a)univ-avignon.fr

cathy.vieillescazes(a)univ-avignon.fr
} 
apparus depuis des siècles, des produits commerciaux d'espèces botaniques différentes. Ainsi les anciennes variétés africaines de gomme ammoniaque provenaient probablement de Ferula spp., et plus particulièrement de l'espèce gummifera (Faure, 1996). Plus récemment, la gomme ammoniaque a été exclusivement désignée comme étant le produit d'exsudation des Dorema (Dorema ammoniacum) (Frocrain, 1994).

Les difficultés rencontrées quant à l'interprétation des textes anciens, la complexité de structures chimiques souvent voisines associées à des quantités de matière souvent infimes nous ont conduit à utiliser l'outil chromatographique $\mathrm{CPG} / \mathrm{SM}$ (Chromatographie en Phase Gazeuse couplée à la Spectrométrie de Masse).

\section{Technique et échantillons}

\subsection{Description des échantillons archéologiques}

Les échantillons possèdent la même origine : ils furent prélevés lors des fouilles effectuées par J. de Morgan dans les années 1894-1895 sur le site de Dashour.

L'échantillon référencé L24 provient du mobilier de la sépulture de la princesse Sat-Hathor, fille de Sésostris II, XII ${ }^{\text {èm }}$ dynastie (1897-1844 av. J.-C.). Ce prélèvement envoyé à Victor Loret fut répertorié grâce aux indications des hiéroglyphes comme l'association d'un premier groupe signifiant "un parfum de premier choix» et d'un second représentant un végétal. L'huile-onguent faisait partie des neuf huiles rituelles des funérailles jusqu'à la basse époque.

L42 provient du tombeau d'une "fille royale" nommée Khnoumit. Plus précisément, il fut prélevé au sein d'un récipient dans l'enceinte de la pyramide du pharaon Amenemhat II, troisième souverain de la XII ${ }^{\mathrm{cme}}$ dynastie (1929-1892 av. J.-C. ou 1994-1797 av. J.-C.). Cependant Khnoumit comme ses consœurs ensevelies dans le même ensemble funéraire n'a en aucun cas pu être rattachée à la famille royale. Les éléments d'information sur la recette de fabrication reflètent une composition à base d'huile végétale, de graines, de parfum au lotus blanc et d'un produit non identifié pouvant correspondre à une résine végétale.

Quant au prélèvement L36, il provient du mobilier de la "princesse" Ita, sceur de Khnoumit. Sa provenance initiale est identique, c'est-à-dire l'enceinte de la pyramide d'Amenemhat II. La suscription relevée donne l'attribution à un "onguent d'exaltation". Selon le laboratoire d'Edfou, cet échantillon ne serait pas un produit naturel mais un mélange de graisse de canard ou d'oie et d'aromates.

\subsection{Résines commerciales et molécules standards}

Cette étude porte notamment sur des résines de la famille des Ombellifères: deux échantillons de galbanum (Ferula galbaniflua) d'origine commerciale différente (Encens du monde - Asie Concept, Castelnau-lelez, France et Anselme, Marseille, France), ainsi qu'une gomme ammoniaque dont l'appartenance botanique n'est pas clairement définie (Anselme, Marseille, France).
En ce qui concerne les standards commerciaux utilisés comme molécules de référence, deux composés coumariques, l'ombelliférone ou 7-hydroxycoumarine (Extransynthèse, Genay, France) et la 4-méthylombelliférone (Fluka A.G., Nuchs, Suisse) et des dérivés diterpéniques à squelette abiétane et pimarane (Helix Biotech, Richmond B.C., Canada) ont été analysés (tab. 1).

\subsection{Préparation des échantillons}

$5 \mathrm{mg}$ d'échantillons sont triméthylsilylés avec une solution constituée de $0,5 \mathrm{ml}$ de pyridine anhydre, $0,45 \mathrm{ml}$ d'hexaméthyldisilazane (HMDS) et $0,3 \mathrm{ml}$ de triméthylchlorosilane (TMSCl). La réaction est effectuée à température ambiante pendant $30 \mathrm{~min}$, temps au bout duquel la solution est évaporée à sec sous courant d'azote ou d'argon avec un chauffage inférieur à $40^{\circ} \mathrm{C}$. Le résidu ainsi obtenu est alors solubilisé dans $0,6 \mathrm{ml}$ d'éther éthylique de grade analytique (Merck), puis directement injecté en CPG/SM.

\subsection{Conditions d'analyse chromatographique}

Les analyses en chromatographie en phase gazeuse ont été réalisées à l'aide d'un chromatographe Varian Saturn 3900, équipé d'un injecteur Varian 1177 et couplé à un spectromètre de masse équipé d'un analyseur de type piège ionique, Varian $2100 \mathrm{~T}$. La colonne capillaire utilisée possède une longueur de $30 \mathrm{~m}$, un diamètre interne de $0,25 \mathrm{~mm}$ et une épaisseur de film de $0,25 \mu \mathrm{m}$ de $5 \%$ phényl, $95 \%$ diméthylsiloxane : il s'agit d'une CP-Sil 8 CB Low Bleed/MS (Varian). La température initiale du four est de $50^{\circ} \mathrm{C}$, pendant $2 \mathrm{~min}$, puis il y a une augmentation de celle-ci de $8^{\circ} \mathrm{C} / \mathrm{min}$ jusqu'à $250^{\circ} \mathrm{C}$, suivi d'une deuxième élévation de température à $3^{\circ} \mathrm{C} / \mathrm{min}$ jusqu'à $350^{\circ} \mathrm{C}$. L'injecteur, la trappe ainsi que la ligne de transfert sont maintenus respectivement à 250,200 et $300^{\circ} \mathrm{C}$. Le temps d'analyse est de 60 minutes. Les échantillons sont injectés $(1 \mu l)$ en mode split dont le rapport est de 20 . Un débit continu de $1 \mathrm{ml} / \mathrm{min}$ d'hélium de grade analytique est utilisé.

\begin{tabular}{|c|c|}
\hline Composé standard & Temps de rétention (min) \\
\hline ombelliférone & 21,9 \\
\hline 4-méthylombelliférone & 23,9 \\
\hline rétène & 26,6 \\
\hline acide pimarique & 27,4 \\
\hline acide sandaracopimarique & 27,7 \\
\hline acide isopimarique & 27,9 \\
\hline acide déhydroabiétique & 28,4 \\
\hline acide abiétique & 28,78 \\
\hline
\end{tabular}

Tableau 1 : Temps de rétention des composés standards. Table 1: Retention time of reference compound. 
Le voltage du multiplicateur d'électron est à $1400 \mathrm{~V}$, le temps d'ionisation dure $25000 \mu$ s et il s'effectue par impact électronique. Le détecteur scanne des masses comprises entre 40 et $650(\mathrm{~m} / \mathrm{z})$ avec un voltage ionisant de $70 \mathrm{eV}$. L'identification des acides gras a été réalisée à partir de la banque de données de spectres de masse NIST'98.

\section{Résultats et discussion}

Les oléo-gommo-résines de la famille des Ombellifères (syn. Apiacées) sont définies comme étant des produits d'exsudation spontanés ou provoqués (Perrot, 1943). Elles sont sécrétées à partir d'un certain nombre d'Ombellifères appartenant aux genres botaniques Ferula et Dorema; elles sont constituées par une émulsion naturelle d'huiles essentielles et de résines en présence de gomme (polysaccharides). Toutes les sécrétions des férules se caractérisent par la présence d'une molécule à squelette coumarique : l'ombelliférone qui est absente de la gomme ammoniaque Dorema ammoniacum (Frocrain, 1994). Cette molécule possède un caractère hautement spécifique dans le sens où elle est absente des autres espèces végétales. Il s'agit donc d'un véritable biomarqueur du genre Ferula.

Une étude préliminaire par CPG/SM a permis de caractériser des résines commerciales contemporaines d'Ombellifères. L'analyse des deux spécimens de galbanum traduit la présence de traces d'ombelliférone, tandis que dans l'échantillon étiqueté gomme ammoniaque sont présentes à la fois l'ombelliférone, en quantité non négligeable et la 4-méthylombelliférone, en faible proportion (fig. 1). Ceci permet de conclure que ce dernier n'est pas une gomme ammoniaque au sens actuel du terme (prove-

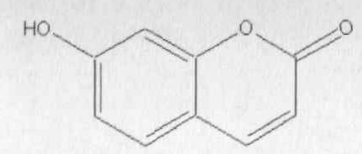

(a)

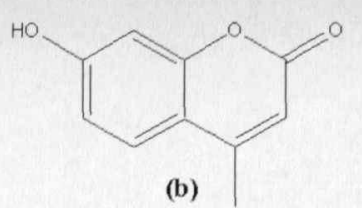

(b)
Figure 1 : Structures chimiques de l'ombelliférone (a) et de la 4méthylombelliférone (b).

Figure 1: Chemical structures of umbelliferone (a) and 4-methylumbelliferone (b).

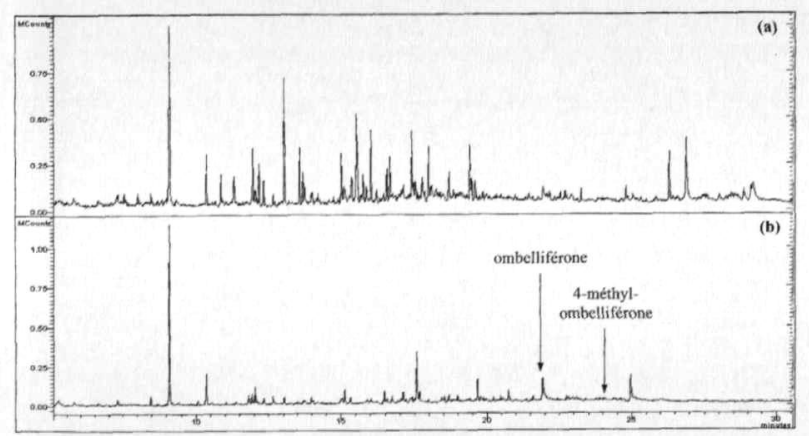

Figure 2 : Chromatogrammes RIC de deux espèces contemporaines commerciales de férules ((a) Ferula galbaniflua et (b) gomme ammoniaque (Ferula)).

Figure 2: RIC chromatogram of two fresh ferula species ((a) Ferula galbaniflua et (b) ammoniac gum (Ferula)). nant de Dorema) mais issu d'une Ferula sp. Il est donc possible de distinguer chimiquement ces deux espèces de férules, d'une part en fonction de la proportion d'ombelliférone et d'autre part à partir de la présence ou de l'absence de son homologue méthylé en position 4 (fig. 2).

Ces résultats ont pu notamment être appliqués à l'étude de l'échantillon archéologique référencé L24. En effet, le chromatogramme obtenu par chromatographie en phase gazeuse traduit la présence de deux molécules à noyau coumarique correspondant respectivement à l'ombelliférone et à la 4-méthylombelliférone. Ces deux composés sont observés en proportion non négligeable et traduisent la présence d'une résine appartenant à la famille des Ombellifères et plus précisément au genre Ferula.

La présence de glycérol et d'acides gras (saturés), constituants des triglycérides, a été également identifiée au sein de ce prélèvement (fig. 3). Les compositions qualitatives et quantitatives des différentes huiles ou graisses peuvent être significatives, compte-tenu de leur nature, animale ou végétale (Karleskind, 1992). Cependant, il n'en est pas de même pour des corps gras dégradés, soit naturellement, soit de manière anthropique. En effet, les acides gras saturés sont relativement stables au cours du temps, ce qui n'est pas le cas des corps gras insaturés. Ces acides se dégradent d'autant plus que leur nombre d'insaturations est grand. Les acides gras polyinsaturés sont si sensibles aux phénomènes d'oxydation, qu'ils sont rarement retrouvés (Evershed, 1992). Lors de la dégradation des graisses, les acides gras insaturés tendent à disparaître et sont remplacés par des acides gras saturés contenant deux atomes de carbone en moins (Tchapla et al., 1999). Il faut donc être critique vis-à-vis des interprétations à donner quant à l'origine biologique du corps gras étudié. Le rapport des quantités d'acides gras saturés a souvent été utilisé comme critère d'identification de l'origine d'un corps gras (Mills \& White, 1994 ; Bourgeois et Marquet, 1992). Les rapports des quantités acide palmitique / acide stéarique (C16:0 / C18:0) et acide palmitique/acide myristique (C16:0 / C14:0) ont été employés pour caractériser la présence d'un corps gras végétal ou animal.

Les divers rapports de quantités en acides gras saturés observés au sein de l'échantillon archéologique L24 correspondent à $\mathrm{C} 16: 0 / \mathrm{C} 18: 0=2,7$ et $\mathrm{C} 16: 0 / \mathrm{C} 14: 0=30,5$. L'interprétation prudente de ces résultats pourrait nous amener à conclure à la présence d'un corps gras d'origine animale, de type graisse de canard.

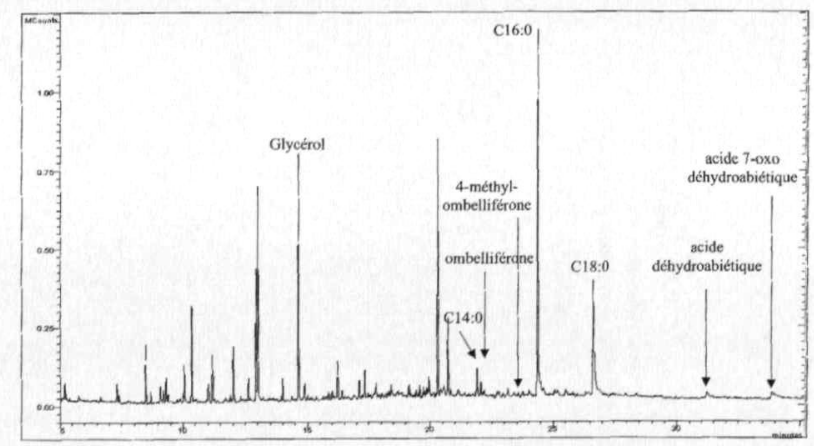

Figure 3 : Chromatogrammes RIC de l'échantillon archéologique L24. Figure 3: RIC Chromatogram of L24 archaeological sample. 
Une autre famille de composés a été identifiée dans ce prélèvement, il s'agit de dérivés diterpéniques à squelette abiétane : les acides déhydro- et 7-oxodéhydroabiétique. Ces composés proviennent de la dégradation de l'acide abiétique qui est caractéristique des résines appartenant à l'ordre des Conifères, plus précisément à la famille des Pinaceae. Ce résultat est peu surprenant, compte-tenu de la présence relativement fréquente de ces molécules au sein d'échantillons archéologiques (Régert et Rolando, 2002 ; Serpico, 1995 ; Evershed et al., 1985 ; Weser et al., 1998). Par contre, il est beaucoup plus original d'identifier une formulation correspondant à un mélange complexe constitué d'un corps gras et de deux résines végétales différentes (Mathe et al., 2003).

Deux autres échantillons archéologiques référencés L42 et L36 ont été également analysés par CPG/SM. Les résultats obtenus traduisent de grandes similitudes; en effet, les deux chromatogrammes sont parfaitement superposables, seule l'intensité relative de l'ensemble des pics diffère (fig. 4). Concernant les constituants de ces mélanges, des diterpènes à squelette abiétane et pimarane ont pu être identifiés avec assurance. Bien que la plupart des acides diterpéniques contenus dans les résines aient la même masse, ils sont souvent faciles à différencier en spectrométrie de masse, car la localisation des doubles liaisons oriente les mécanismes de fragmentation (Audier et al., (a) et (b) 1966 ; Enzell et Wahlberg, 1969). Par ordre d'élution, les composés rencontrés sont le rétène et les acides sandaracopimarique, isopimarique, déhydroabiétique, abiétique (traces) et 7-oxodéhydroabiétique (traces) (fig. 5). Comme précédemment ces dérivés sont présents au sein des résines de Conifères, mais ne permettent pas de caractériser avec précision la famille et l'espèce de la matière résineuse détectée. Sous l'influence de la préparation de l'échantillon et/ou de sa dégradation au cours du temps, les biomarqueurs des résines fraîches ne sont pas toujours conservés dans les échantillons archéologiques d'où la difficulté d'identifier précisément l'origine botanique des matériaux résineux employés.

\section{Bilan}

Tous les lots d'échantillons archéologiques, parfaitement répertoriés dès réception par $\mathrm{V}$. Loret, forment un ensemble homogène et intéressant à plus d'un titre. Les

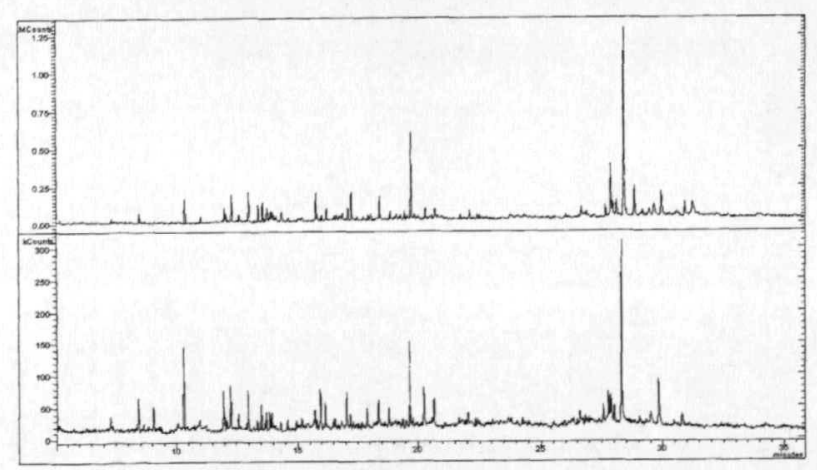

Figure 4 : Comparaison des chromatogrammes RIC des échantillons archéologiques L42 et L36.

Figure 4: Comparison of RIC chromatogram of $L 42$ and $L 36$ archaeological samples.

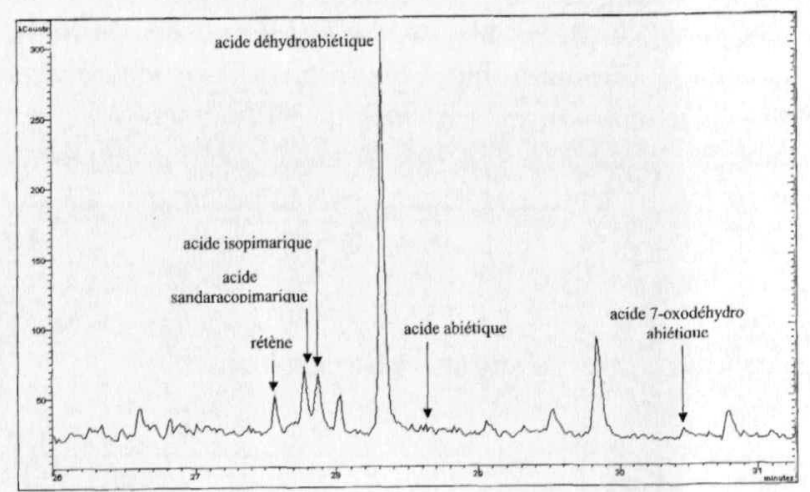

Figure 5: Agrandissement de la zone diterpénique du chromatogramme RIC de l'échantillon L42.

Figure 5: Zoom of diterpenic area of RIC chromatogram of L42 sample.

éléments d'information à leur sujet sont essentiels car ils correspondent à des mélanges complexes selon les listes d'Edfou ou de Dendera.

Le prélèvement L24 a été identifié comme étant un mélange d'un corps gras et de deux résines végétales, la première appartenant à la famille des Conifères et la seconde aux Ombellifères, plus précisément au genre Ferula. Cette composition est en accord avec le texte en hiéroglyphes évoquant une huile-onguent à base de végétaux.

Bien que la suscription hiéroglyphique des prélèvements L42 et L36 soit différente, ces échantillons traduisent une composition chimique identique. Cependant ni la présence d'un corps gras, ni celle d'une résine triterpénique n'ont été mises en évidence, ce qui ne permet pas en l'état de confirmer l'hypothèse d'une composition. Un élément est cependant acquis, ces échantillons contiennent une résine de Conifère.

L'outil analytique contribue à mieux cerner la composition chimique de mélanges parfois très complexes, en l'occurrence de nature archéologique. L'apport de la $\mathrm{CPG} / \mathrm{SM}$ a ainsi permis de corroborer et de compléter les résultats obtenus sur ces mêmes échantillons par CLHP/ détection fluorimétrique (Martin et al., 2001).

Les informations recueillies sont précieuses car elles fournissent d'importants renseignements sur les habitudes d'une époque ou sur les recettes jadis employées.

\section{Remerciements}

Les auteurs expriment leur gratitude au Professeur J.C. Goyon (Professeur émérite de l'Université de Lyon II) pour leur avoir permis d'accéder aux échantillons de la collection V. Loret, et pour les précieuses informations sur les données archéologiques qui s'y rapportent.

\section{Bibliographie}

AUDIER, H. E., BORY, S., DEFAYE, G., FÉTIZON, M. et MOREAU, G., 1966 (a) - Spectres de masse des terpènes. II Influence du noyau aromatique sur la fragmentation des diterpènes. Bulletin de la société chimique de France, 10, 3181-3186.

AUDIER, H. E., BORY, S., FÉTIZON, M. et ANH, T., 1966 (b) Spectres de masse de terpènes. III - Influence de la liaison éthylénique sur la fragmentation des diterpènes. Bulletin de la société chimique de France, 12, 4002-4010. 
BOURGEOIS, G. et MARQUET, J.-C., 1992 - Des traces de graisses animales sur le site néolithique final du Petit Paulmy à Abilly (Indre et Loire), Bulletin de la Société Préhistorique Française, 89, 47-49.

ENZELL, C. R. et WAHLBERG, I., 1969 - Mass spectrometric studies of diterpens. 6 Aromatic diterpenes, Acta Chemica Scandinavica, 23, 871-891.

EVERSHED, R. P., JERMAN, K. and EGLINTON, G., 1985 - Pine wood from the Mary Rose, Nature, 314, 528-530.

EVERSHED, R. P., 1992 - Chemical composition of a bog body adipocere, Archaeometry, 34, 253-265.

FAURE, P., 1996 - Parfums et aromates de l'Antiquité, éd. Fayard, collection Pluricl, 30-35.

FROCRAIN, I., 1994 - Les gommo-oléurésines des Ombellifêres : ase-fétide, galbanum et gomme ammoniaque, thèsc de pharmacic, Université de Paris V.

KARI.ESKIND, A., 1992 - Manuel des corps gras, ed. Tec et Doc, Paris, chap. II.

MARTIN, P., ARCHIER, P., VIEILIESCAZES, C. and PISTRE, M.S., 2001 - HPLC coupled with fluorimetric detection for the identification of natural resins in archaeological materials. Chromatographia, 53, 7/8, 380-384

MATHE, C., ARCHIER, P., CULIOLI, G. et VIEILLESCAZES, C., 2003 - Caractérisation chimique d'une résine naturclle en Egypte ancienne : application à un cxcmplc de la collection Victor Loret, Revue d'Archéométrie, 27, 43-47.
MILLS, J. S. and WHITE, R., 1994 - The Organic: Chemistry of Museum Objects, Butterworth-Heinemann, Ox ford, 171-172.

PERRO'T, E., 1943 - Matières premières usuelles du règne végétal, tome II, Masson \& Cic éditcur, Paris, 1624-1681.

RÉGERT, M. et ROLANDO, C., 2002 - Identification of archacological adhesives using direct inlet clectron ionization mass spectrometry, Analytical Chemistry, 74, ${ }^{\circ} 5,965-975$.

SERPICO, M., 1995 - Chemical analysis of coniferous resins from ancient Egypt using Gas Chromatography/Mass Spectrometry (GC/ MS), $7^{\text {th }}$ International Congress of Egyptologists, Cambridge, 3-9 september 1995, Oxbow Books, 163-164.

TCHAPLA, A., BLETON, J., GOURSAUD, S. et MÉJANELI,E, P., 1999 - Contribution à la connaissance des substances organiques utilisćes en Egypte ancienne. L'apport de techniques physico-chimiques d'analyse. Dans : Encyclopédie religicuse de l'Univers végétal - Croyances phytoreligieuses de l'Egypte ancienne, OrMonsp X, 1, 445-488.

WESER, U., KAUP Y., ETSPÜLER, H., KOLLER, J. and BAUMER, U., 1998 - Embalming in the old kingdom of pharaonic Egypt, Analytical Chemistry News \& Features, 511-516. 